\title{
Vogt-Koyanagi-Harada disease: headache as an initial manifestation
}

\author{
Mustafa Tavsanli · Derya Uluduz $\cdot$ Sabahattin Saip · \\ Gurhan Kendiroglu
}

Received: 29 March 2008/Accepted: 12 May 2008/Published online: 30 May 2008

(C) Springer-Verlag 2008

Vogt-Koyanagi-Harada (VKH) disease is a granulamatous inflammatory disease affecting the eyes, auditory system, meninges and the skin [1]. Although headache, a prodromal sign, is present in $67 \%$ of patients [2], VKH disease is rarely encountered in neurology practice [3, 4].

Case 1 A 22-year-old woman reported a sudden onset of continuous severe headache followed by gradually evolved bilateral blurred vision. An MRI of the brain and orbital were normal. Analysis of CSF revealed monocytosis. Neuroophthalmologic examination revealed swelling of the optic disks and a cell reaction in vitreous and anterior chamber (Fig. 1a, b). She was diagnosed as incomplete VKH disease. For 10 days, $1 \mathrm{~g} /$ day intravenous methyl prednisolone (IVMP) treatment was administered, following an oral dose with $2 \mathrm{mg} / \mathrm{kg}$ per day. One week later, her symptoms were completely improved. Steroid treatment continued for 3 months with gradual decrease.

Case 2 A 47-year-old woman presented with a 3-week history of diffuse and bilateral, orbito-retroorbital headache and bilateral acute vision loss. Cranial CT, MRI and MR angiography were normal. CSF pressure was normal.
Analysis of CSF revealed $90 \%$ of monocytosis. Her funduscopic examination showed hyperemia and swelling of the optic disk. She was diagnosed with incomplete VKH. For ten days, $1 \mathrm{~g} / \mathrm{kg}$ IVMP treatment was administered. In the sixth day of the treatment headache and visual complaints were improved. Treatment continued with high dose oral steroid $(2 \mathrm{mg} / \mathrm{kg})$ and decreased gradually.

In patients with VKH disease meningeal involvement may cause severe devastating headache mimicking other types of acute headaches. Symptoms might be interpreted as optic neuritis, pituitary apoplexy or intracranial hypertension if headache is associated with ocular findings. Moreover, integumentary symptoms such as allopesia, poliosis, vitiligo appear only late at the convalescent stage of the disease. Therefore, the diagnostic criteria lack sensitivity for the early diagnosis of VKH disease particularly in neurology emergencies. If the brain MRI is normal, diagnosis could be more confusing. VKH disease should be considered in the differential diagnosis of patients presenting with acute headache followed by bilateral blurred vision. Early diagnosis and adequate treatment are the keys to success. Corticosteroid is essential in the management of VKH disease.

M. Tavsanli $(\bowtie) \cdot$ D. Uluduz · S. Saip

Department of Neurology,

Cerrahpasa Medical School,

Istanbul, Turkey

e-mail: drtavsanli@yahoo.com

G. Kendiroglu

Department of Neuroophthalmology,

Dunya Goz Hospital,

Istanbul, Turkey 


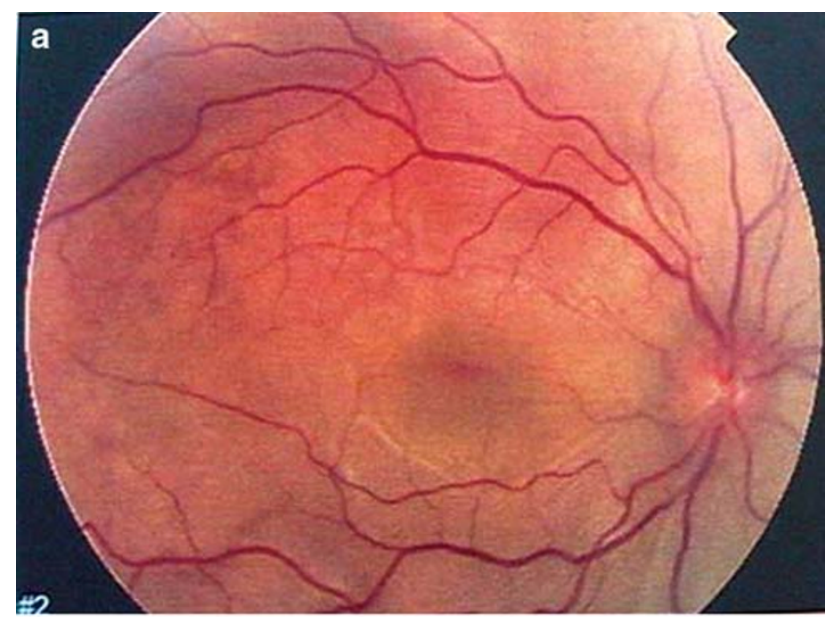

\section{References}

1. Sugiura A (1978) Vogt-Koyanagi-Harada disease. Jpn J Ophthalmol 22:9-35

2. Cho HJ, Ahn YJ, Byeon SH et al (2008) Thunderclap headache as initial manifestation of Vogt Koyanagi Harada disease. Headache 48:153-155

3. Mantovani A, Resta A, Herbort CP et al (2007) Work up, diagnosis and management of acute Vogt Koyanagi Harada disease. Int Ophthalmol 27:105-115

4. Read R, Rao NA, Cunningham ET (2000) Vogt Koyanagi Harada disease. Curr Opin in Ophthalmol 11:437-442

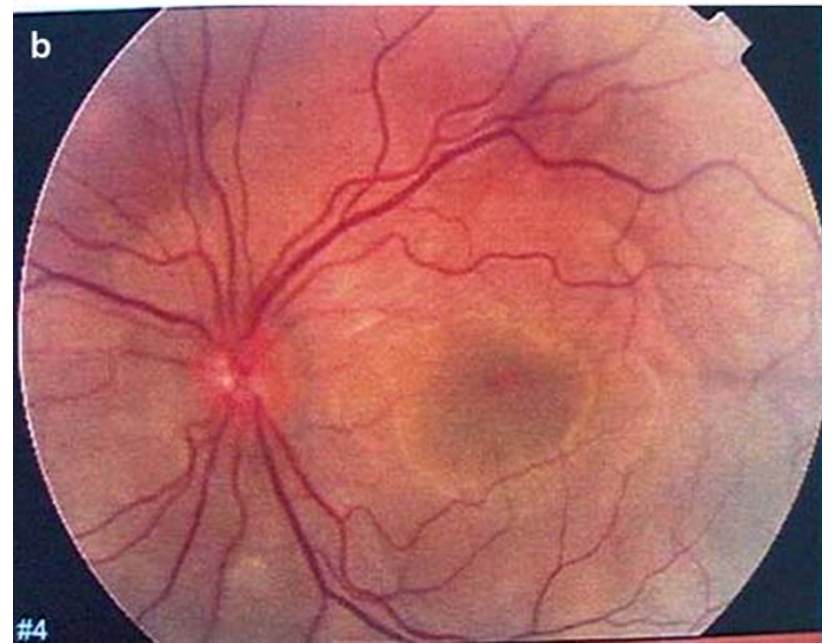

Fig. 1 a Pathologic funduscopic examination with papilledema and cell reaction in vitreous and anterior chamber. b Pathologic funduscopic examination with papilledema and cell reaction in vitreous and anterior chamber 